\title{
Effect of walking surface perturbation training on slip propensity and local dynamic stability
}

\author{
Jian $\operatorname{Liu}^{\mathrm{a},{ }^{*}}$ and Sukwon Kim ${ }^{\mathrm{b}}$ \\ ${ }^{a}$ Department of Health and Human Performance, University of Houston, 104GAR, 3855 Holman St., Houston, TX, \\ 77204-6015, USA \\ ${ }^{\mathrm{b}}$ Department of Physical Education, Chonbuk National University, Jeonju, South Korea
}

\begin{abstract}
The objective of the current study was to evaluate the effect of walkway perturbation training on human dynamic walking stability and slip propensity. A new walkway perturbation training program was developed using an instrumented, split-belt treadmill. Walkway surface perturbation was composed of multiple, randomized perturbation pulses. Each pulse was created by sudden accelerating the treadmill belt underneath the dominant foot briefly. Five healthy older adults were involved in a laboratory study. An inertial measurement unit was attached to the subject's low back region to record 3D acceleration. Maximum Lyapunov exponent (maxLE) and transitional acceleration of whole body center of mass (TA_COM) were computed to quantify the local dynamic stability and slip propensity, respectively. The results indicated a significant training effect on both maxLE and TA_COM. It was concluded that the walkway perturbation training program significantly improved local dynamic stability and reduced slip propensity.
\end{abstract}

Keywords: fall prevention, perturbation training, acceleration, dynamic stability

\section{Introduction}

Fall accidents continue to be a serious threat to the health of the elderly. In 2007 , over 18,000 older adults died from falling [9]. In 2009, 2.2 million older adults visited emergency departments for nonfatal fall injuries [1]. To that end, developing innovative fall intervention strategies to prevent falls among older adults has been categorized as Tier 1 research priority by CDC [9]. Recently, a novel perturbationtraining paradigm emerged, suggesting that an individual can adaptively enhance his/her stability [10]. This paradigm is based on the motor learning theory that an individual's own neuromuscular and reflexive protective mechanisms against balance loss can be developed or enhanced with repeated perturbations [11]. However, the efficacy of such training still needs to be established. Therefore, the objective of the current study was to evaluate the effect of the walkway perturbation training on human dynamic walking stability and slip propensity. It was hypothesized that after walkway perturbation training, an individual would improve his/her local dynamic stability and reduce his/her slip propensity.

\section{Methods}

Five female older adults (mean/SD, age: 71.4/2.6 years; weight: $63.7 / 8.7 \mathrm{~kg}$; height: $163.7 / 4.9 \mathrm{~cm}$ ) were involved in a laboratory study. All the participants were healthy and free from major musculoskeletal disorders. The study protocol was approved by the Committee for Protection of Human Subjects at University of Houston. Informed consent was obtained from each subject. The experiment was composed of three sessions: baseline, perturbation training, and assessment. During the baseline and assessment sessions, one inertial measurement unit (IMU, Xsens, The Netherlands) was attached to the participant's low back region (i.e., L5/S1) to measure 3D

*Corresponding author. E-mail: jliu30@uh.edu ,Tel: 713-743-9045, Fax: 713-743-9860 
acceleration at a sampling of $100 \mathrm{~Hz}$. Each participant walked on an instrumented split-belt treadmill at his/her self-selected speed (mean/SD: $1.2 / 0.2 \mathrm{~m} / \mathrm{s}$ ). One 2-min trial of low back 3D acceleration was taken in both baseline and assessment sessions. During the perturbation training session, 16-min walking with surface perturbations was introduced to each participant. Walkway surface perturbation was composed of multiple, randomized perturbation pulses. Each perturbation pulse was created by sudden accelerating $\left(5 \mathrm{~m} / \mathrm{s}^{2}\right)$ the treadmill belt underneath the dominant foot for $0.5 \mathrm{~s}$. Each perturbation was induced at the time of heel contact, which was determined by the embedded force plate (i.e., vertical force exceeds $7 \mathrm{~N}$ ). The time interval between consecutive perturbation pulses was randomized between 1 to 5 gait cycles.

Local dynamic stability, as quantified by maximum Lyapunov exponent (maxLE), was calculated from the 2-min vertical low-back acceleration using published method $[6,8]$. Slip propensity was quantified by transitional acceleration of whole body center of mass (TA_COM), which was determined as the anterio-posterior low-back acceleration at the time of

(a)

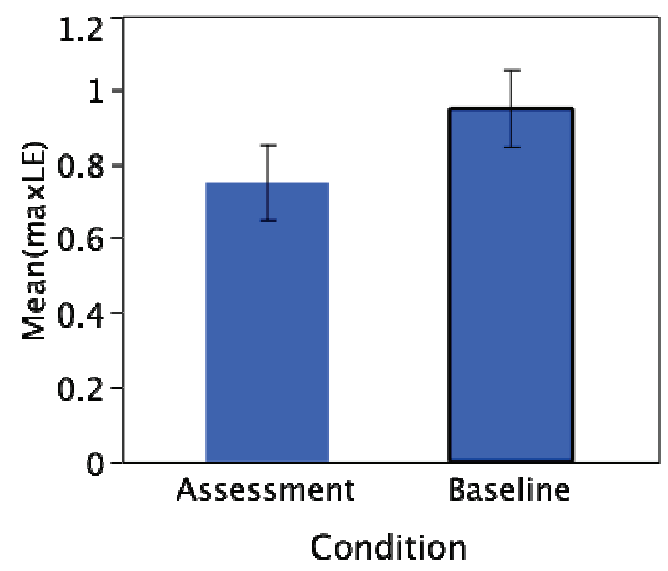

heel contact [7]. One-way within-subject ANOVA was performed in JMP 8.0 (SAS Institute Inc., Cary, $\mathrm{NC}$ ), with one independent variable (condition: baseline and assessment) and two dependent variables (maxLE and TA_COM). The significant level was set as $\alpha=0.05$.

\section{Results}

There was a significant condition effect on both $\operatorname{maxLE}(\mathrm{p}=0.0070)$ and TA_COM $(\mathrm{p}=0.0465)$. Specifically, there was a $21 \%$ decrease in maxLE during the assessment session than during the baseline session (Figure 1a), which indicated an improved local dynamic stability after the walkway perturbation training. In addition, there was a $62.5 \%$ increase in TA_COM during the assessment session than during the baseline session (Figure 1b), which indicated a reduced slip propensity after the walkway perturbation training. (b)

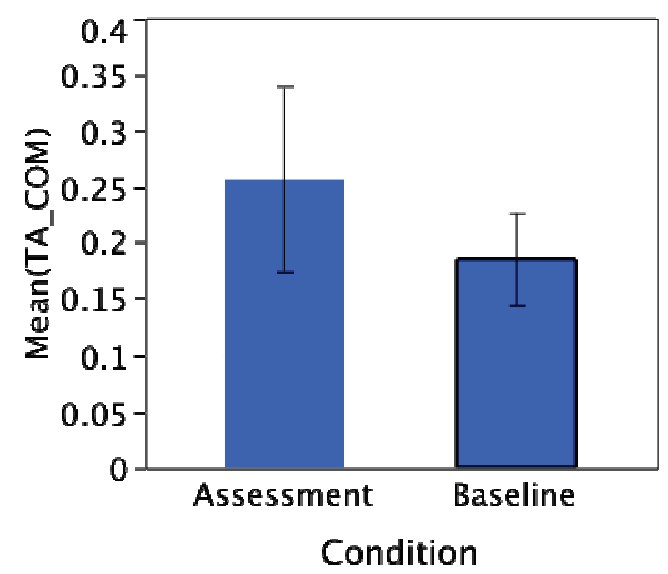

Figure 1 - Mean maxLE and TA_COM by condition. Error bar indicates 1 standard error.

\section{Discussions}

The current study aimed to evaluate the effect of the walkway perturbation training on human dynamic walking stability and slip propensity. The study results supported both hypotheses that after walkway perturbation training, an individual significantly im- proved his/her local dynamic stability and reduced his/her slip propensity. Although the concept of perturbation training is not new, the current study presented a new method to induce walkway perturbation training, which utilized the unique feature of an instrumented split-belt treadmill. Through further development and validation, it is anticipated that such training method and instrument can be incorporated 
into an effective fall prevention program for community dwelling elderly.

The concept of local dynamic stability has been utilized in many different aspects of human movement studies $[2,3]$. Our previous research has demonstrated that local dynamic stability could differentiate fall-prone elderly from healthy counterparts $[6$, $8]$. The results from the current study showed that local dynamic stability could also be used as a sensitive measure to evaluate the effectiveness of a fall prevention program.

The walkway perturbation training in the current study also reduced the risk of slipping as evidenced by a significant increase in TA_COM. TA_COM has been utilized as an important biomechanical parameter in slips and falls research since 2003 [7]. A significant and strong linear correlation $[4,7]$ has been found between TA COM and the required coefficient of friction, which is a direct indicator of slip propensity. Slower whole body COM transfer (i.e., higher TA_COM) was also regarded as a contributing factor for slip-induced falls [7], as higher TA_COM could help individuals to retract their slipping foot or progress their COM forward closer to slipping foot [5]. Therefore, the current results may implicate that the training program could enhance the participants' capability to recover from slip-induced falls.

As a pilot investigation, the current study is facing various limitations, which include limited sample size and homogenous participant population (i.e., healthy female elderly). In addition, retention effect also warrants further investigation for any types of training program. To what degree that the perturbation training effect can be transferred to situations such as slip-induced falls in daily life also needs further exploration.

In summary, a new walkway perturbation training program is presented in the current study. It has been demonstrated that this training could significantly improve the local dynamic stability and reduce the slip propensity.

\section{References}

[1] Center for Disease Control and Prevention. (2010). Webbased injury statistics query and reporting system (WISQARS): National Center for Injury Prevention and Control. Accessed 11/30/2010

[2] Dingwell, J. B., Robb, R. T., Troy, K. L., \& Grabiner, M. D. (2008). Effects of an attention demanding task on dynamic stability during treadmill walking. Journal of Neuroengineering and Rehabilitation, 5.

[3] Dingwell, J. B., \& Kang, H. G. (2007). Difference between local and orbital dynamic stability during human walking. Journal of Biomechanical Engineering-Transactions of the Asme, 129(4), 586-593.

[4] Kim, S., Lockhart, T., \& Yoon, H. Y. (2005). Relationship between age-related gait adaptations and required coefficient of friction. Safety Science, 43(7), 425-436.

[5] Kim, S., \& Lockhart, T. (2010). Effects of 8 weeks of balance or weight training for the independently living elderly on the outcomes of induced slips. International Journal of Rehabilitation Research, 33(1), 49-55.

[6] Lockhart, T. E., \& Liu, J. (2008). Differentiating fall-prone and healthy adults using local dynamic stability. Ergonomics, 51(12), 1860 - 1872.

[7] Lockhart, T. E., Woldstad, J. C., \& Smith, J. L. (2003). Effects of age-related gait changes on the biomechanics of slips and falls. Ergonomics, 46(12), 1136-1160.

[8] Liu, J., Lockhart, T. E., Jones, M., \& Martin, T. (2008). Local Dynamic Stability Assessment of Motion Impaired Elderly Using Electronic Textile Pants. IEEE Transactions on Automation Science and Engineering, 5(4), 696-702.

[9] National Center for Injury Prevention and Control. (2009). CDC Injury Research Agenda, 2009-2018

[10]Pai, Y. C., \& Bhatt, T. S. (2007). Repeated-slip training: An emerging paradigm for prevention of slip-related falls among older adults. Physical Therapy, 87(11), 1478-1491.

[11]Pavol, M. J., Runtz, E. F., \& Pai, Y. C. (2004). Young and older adults exhibit proactive and reactive adaptations to repeated slip exposure. Journals of Gerontology Series aBiological Sciences and Medical Sciences, 59(5), 494-502. 